\title{
Retrospective Study on the Safety and Efficacy of Clopidogrel in the Treatment of Acute Cerebral Infarction
}

\author{
Xiufeng Xin, Anding Xu*, Wanyong Yang, Zefeng Tan \\ Department of Neurology and Stroke Center, The First Affiliated Hospital of Jinan University, Institute of Clinical Neuroscience, Guangzhou, \\ China
}

\author{
Email address: \\ tlil@jnu.edu.cn (Anding Xu) \\ ${ }^{*}$ Corresponding author
}

\section{To cite this article:}

Xiufeng Xin, Anding Xu, Wanyong Yang, Zefeng Tan. Retrospective Study on the Safety and Efficacy of Clopidogrel in the Treatment of Acute Cerebral Infarction. International Journal of Neurologic Physical Therapy. Vol. 4, No. 1, 2018, pp. 24-28.

doi: $10.11648 /$ j.ijnpt.20180401.14

Received: April 6, 2018; Accepted: April 23, 2018; Published: May 17, 2018

\begin{abstract}
Till now there's no large sample, randomized and double-blinded research of clopidogrel in acute cerebral infarction. There have been some studies of combined and loading dosage of clopidogrel for antiplatelet treatment but the NIHSS is no more than 5 potints. Our study is to evaluate the efficacy and safety of clopidogrel in acute ischemic stroke. It's a single center retrospective study. Collect information of patients with acute ischemic stroke from January 1st 2012 to May 31st 2015, using of different antiplatelet drugs, occuring of progressive ischemic stroke, risk factors of cardiovascular and cerebrovascular diseases, etiological classification of cerebral infarction, NIHSS scores on admission and 7 days after admission were collected and was calssified into different groups (NIHSS $\leq 3,4-7,8-15$ and $>15$ potints). correlation statistical analysis was performed with chi-square test. A total of 1008 patients were collected, 94 of them had progressive ischemic stroke. There was no significant difference between aspirin group and clopidogrel group (routine clopidogrel group and loading clopidogrel group) within 7 days of onset. Clopidogrel group was superior to aspirin in reducing early recurrence and deterioration within 24 hours while NIHSS on admission was greater than 3 points. PIS has close relation to admission time after onset and severity of clinc. There was no significant difference in the incidence of safety events between aspirin and clopidogrel (routine clopidogrel and loading clopidogrel). Loading dosage of clopidogrel is as safe as the routine group and aspirin group.
\end{abstract}

Keywords: Acute Cerebral Infarction, Clopidogrel, Progressive Ischemic Stroke,

Cerebral Infarction Hemorrhage Transformation

\section{Introduction}

Although early time using of aspirin for antiplatelet therapy can reduce stroke recurrence, but the net benefit of aspirin is relatively low and is useless for progressive ischemic stroke [1, 2]. Clopidogrel can provide an additional of $8.7 \%$ reduction than aspirin in relative risk [3]. A number of antiplatelet drug studies for patients with acute cerebral infarction such as CHANCE [4], SUMMPRIS [5], SOCRATES [6] and CLASS-CHINA [7] had shown exploration and bright prospect of combined and loading dosage of clopidogrel for antiplatelet treatment. LOAD [8] had proved the safety of loading dosage of clopidogrel and aspirin. In the research of SOCRATES [6], Most of the patients with middle cerebral infarction were mild stroke patients (NIHSS score $\leq 5$ points), in which the CHANCE study [9] answered the TIA (ABCD2 score $\geq 4$ points) and the minor stroke (NIHSS score $\leq 3$ points), the superiority of combined and loading dosage of antiplatelet over aspirin alone.

The superiority of clopidogrel over aspirin was not clear in patients of NIHSS with 4-7 points and lack of related clinical research which worth clinical studying. Our center start to use clopidogrel from 2005 and standard operating procedures (SOP) from 2012 require loading dosage of clopidogrel and combination with aspirin for 7 days in patients of NIHSS score of $\leq 7$ points. Patients with intracerebral infarction were treated with loading dosage of clopidogrel. The aim is to evaluate whether clopidogrel is superior to aspirin and whether loading dosage of clopidogrel is superior to aspirin. 


\section{Methods}

\subsection{Design Overview}

This study was based on a single center and retrospective study. All cases were from the Department of Neurology of the first affiliated Hospital of Jinan University. Patients with acute ischemic stroke admitted to hospital within 7 days of onset from January 1st 2012 to May 31st 2015 were collected.

\subsection{Participants}

Over 18 years of age; The diagnosis of cerebral infarction in patients with acute cerebral infarction within 7 days of onset was in accordance with the diagnostic criteria of cerebral infarction in the National guidelines for the Prevention and treatment of Cerebrovascular Disease in 2014 [10] and was confirmed by CT / MRI. Subjects were excluded if they underwent emergency thrombolytic therapy or intravascular therapy; Patients who received anticoagulant drugs within 7 days of admission included low molecular weight heparin (LMWH); Patients with asymptomatic cerebral infarction were found after admission; Patients enrolled in clinical studies; Patients who developed from transient ischemic attack to cerebral infarction after admission; Patients with previous cerebral infarction were treated with antiplatelet drugs in the recent 2 weeks.

\subsection{Methods of Data Collection}

The electronic medical records included "cerebral infarction", "atherosclerotic cerebral infarction", "cardiogenic cerebral embolism", "lacunar infarction" and "cerebrovascular disease" according to the diagnosis of discharge from January 1st 2012 to May 31st 2015. Screen admission and discharge departments one by one, check each electronic case to register the course of hospitalization and related clinical data, and cross-check, modify and supplement the variable data.

NIHSS score $\leq 7$ points: loading dose of clopidogrel 300 $\mathrm{mg}$ (75mg qd from the next day) and aspirin 100mg QD; determined according to specific circumstances. If the patient has previous gastric hemorrhage and other intolerant double antibodiesa history of cerebral infarction, coronary heart disease, They were given aspirin $100 \mathrm{mg} / \mathrm{d}$ temporarily, clopidogrel $75 \mathrm{mg} / \mathrm{d}$ from the second day, and Clopidogrel / aspirin was given according to ESSEN score after 7 days.

NIHSS score $\geq 8$ points, according to the ESSEN score, clopidogrel (first time of $300 \mathrm{mg}$ loading dose and then $75 \mathrm{mg}$ QD) or aspirin 100mg QD was given.

\subsection{Collection of Clinical Data}

Sex, age of patients with BMI, demographic data of smoking and drinking history. The time from onset to admission. NIHSS, mRS and BI scores of admission and after treatment of $7 \mathrm{~d}$, images of patients admitted to hospital without bleeding transformation, such as hemorrhagic transformation of ECASSII. Record the bleeding in patients with bleeding type conversion and location of infarction.

Past medical history, such as hypertension, diabetes, coronary heart disease, atrial fibrillation, valvular heart disease, stroke (cerebral hemorrhage or cerebral infarction).

Pre-hospital medication: antiplatelet drugs (clopidogrel or aspirin) were excluded, anticoagulants (Dabigavin, warfarin, rivastatin, statins) were excluded.

Choice of antiplatelet and Lipid-lowering drugs after admission.

Factors influencing the recurrence or progression of cerebral infarction and the transformation of cerebral infarction hemorrhage: blood pressure at admission, fasting blood glucose, CRP, low density lipoprotein, INR, whether platelets are less than $200 * 10^{9} / \mathrm{L}$, whether there is fever within 7 days of admission, whether the condition of consciousness disorder at admission is a large area of cerebral infarction.

\subsection{Interpretation of Observation Indicators}

Smoking history, drinking history and past medical history, according to the records of the case.

After admission, hyperlipidemia was detected, and hyperlipidemia was defined as: total cholesterol $>5.18 \mathrm{mmol} /$ $\mathrm{L}$, triglyceride $>1.70 \mathrm{mmol} / \mathrm{L}$, low density lipoprotein $>3.37$ $\mathrm{mmol} / \mathrm{L}$.

Large area cerebral infarction: the Chinese guidelines for the Prevention and treatment of Cerebrovascular Disease in 2014 defined as more than one lobe with a diameter of more than $5 \mathrm{~cm}[10]$.

\subsection{Therapeutic Endpoint Events}

\subsubsection{Main Outcome Endpoint Events}

Early recurrence or progression: NIHSS scores after admission to $7 \mathrm{~d}$ were higher than baseline $\geq 2$ points.

\subsubsection{Secondary Therapeutic Endpoint Events}

New Clinical Vascular events: recurrence of Ischemic Stroke (excluding TIA), Myocardial Infarction, Pulmonary Infarction, Deep Venous Thrombosis, other macrovascular events, Vascular death: death due to stroke (ischemic or hemorrhagic, systemic hemorrhage, myocardial infarction, pulmonary embolism, congestive heart failure, sudden death, or arrhythmia). Adverse reactions / severe adverse reactions.

\subsection{Safety Endpoint Events}

\subsubsection{Main Safety Endpoint Events}

Life-threatening hemorrhage: symptomatic intracranial hemorrhage or severe extracranial hemorrhage (hemoglobin reduction $\geq 50 \mathrm{~g} / \mathrm{L}$ );

Hemorrhagic shock; or need blood transfusion $\geq 4$ units of $\mathrm{RBC}$ or equivalent volume of whole blood.

\subsubsection{Minor Security Endpoint Events}

Moderate haemorrhage: GUSTO study [9] defines (with persistent haemorrhage sequelae, or intraocular hemorrhage leading to severe loss of vision; or requiring a transfusion of 2-3 units of RBC or equivalent volume of whole blood; small bleeding events, Refers to bleeding events that do not require blood transfusion or do not cause hemodynamic changes, such 
as small bleeding spots in the skin, positive occult blood in stool, etc.

\subsection{Statistical Methods}

Statistical Analysis of data by SPISS19.0 Software. Compare the early recurrence or progression of patients whih clopidogrel (routine and loading dosage) or aspirin for $7 \mathrm{~d}$. Measurement data adoption by chi-square test, The counting data is expressed by rate or median, All tests are bilateral hypothesis tests, inspection level $\mathrm{a}=0.05$.

\section{Results}

\subsection{General Information}

From January 1st 2012 to May 31st 2015, a total of 1008 cases were recorded. Among them, 94 cases suffered from early recurrence or progression of major end point events (progressive ischemic stroke, PIS), 8 cases died, and 4 cases of secondary end point events occurred. The test efficiency of the statistical analysis was obviously reduced, so only the statistical analysis of the main curative effect events was carried out. Among them, 2 cases were the main safety endpoint events, 38 cases were secondary safety events. The total end point events were 40 cases. There were 668 males and 340 females, 325 patients with a personal history of smoking, 91 patients with a personal history of drinking, 42 patients with massive infarction, 80 patients with disturbance of consciousness at admission. There were 55 cases of somnolence and 25 cases of coma (the general information features see Table 1).

Table 1. The general information.

\begin{tabular}{lllll}
\hline Parameter & Effective number & minimum value & Maximum value & Average value \\
\hline Age & 1008 & 20 & 96 & 65.02 \\
BMI & 606 & .00 & 41.67 & 23.54 \\
NIHSS on admission & 1008 & 0 & 27 & 5.00 \\
mRS on admission & 1007 & 0 & 5 & 2.69 \\
SBP on admission & 1002 & 13 & 248 & 154.32 \\
DBP on admission & 1001 & 5 & 887 & 86.28 \\
Fasting plasma glucose & 989 & 1.00 & 44.500 & 7.32 \\
INR & 940 & .76 & 11.1 & 1.21 \\
H-CRP & 858 & .00 & 244.36 & 12.52 \\
LDL-C & 990 & 0 & 27 & 3.05 \\
NIHSS at $7^{\text {th }}$ day or discharge & 1000 & 0 & 5 & 4.08 \\
mRS at 7 th day or discharge & 1000 & & & 2.19 \\
\hline
\end{tabular}

The total number of patients with clopidogrel was $876(86.9 \%)$. There were 132 cases $(13 \%)$ in aspirin group, 163 cases $(16 \%)$ in routine clopidogrel group and 205 cases $(20 \%)$ in loading clopidogrel group. The total number of aspirin and clopidogrel alone using patients was 500, which was the main object of this data analysis (see figure 1).

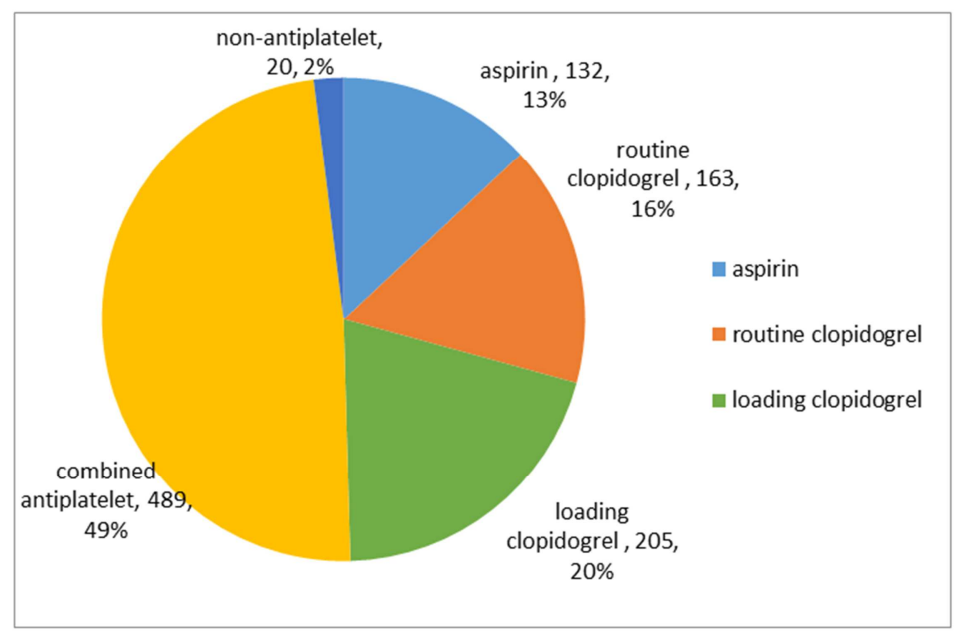

Figure 1. Antiplatelet groups.

\subsection{PIS with Relation to Onset to Hospitalization Time and NIHSS}

Table 2. PIS within or after 24 hours after onset of clinic.

\begin{tabular}{llll}
\hline Group & Cases with PIS & Cases without PIS & Total \\
\hline Admission within 24 hours & $69(13.7 \%)$ & 434 & 503 \\
Admission after 24 hours & $25(4.95 \%)$ & 480 & 505 \\
Total & 94 & 914 & 1008 \\
\hline
\end{tabular}


This table shows that the percentage of progressive ischemic stroke is $13.7 \%$ in the patients within 24 hours from onset to hospitalization and means that over $70 \%$ of the progressive ischemic stroke occurs within the first 24 hours or the first days from onset. The P value is less than 0.001 and means PIS has clear relation to Onset to hospitalization time.

The severity of clinc is classified in groups of NIHSS of 0-3 and over 3 points. There's 233 cases in the $0-3$ group and with 19 cases $(8.15 \%)$ of PIS but without statistical difference among the aspirin and clopidogrel groups (routine and loading dose).

\subsection{PIS with Relation to Different Antiplatelet Groups Within 24 Hours After Onset}

Table 3. PIS between clopidogrel and aspirin groups within 24 hours after onset.

\begin{tabular}{llll}
\hline $\begin{array}{l}\text { Antiplatelet } \\
\text { program }\end{array}$ & Aspirin & $\begin{array}{l}\text { Routine } \\
\text { clopidogrel }\end{array}$ & $\begin{array}{l}\text { Loading } \\
\text { clopidogrel }\end{array}$ \\
\hline Without PIS & 39 & 66 & 83 \\
With PIS (\%) & $19(32.75 \%)$ & $11(14.28 \%)$ & $12(12.63 \%)$ \\
$P$ & 0.045 & 0.057 & 0.021 \\
Total & 58 & 77 & 95 \\
\hline
\end{tabular}

This table shows that PIS is much higher in aspirin group than the routine and loading clopidogrel groups in patients within 24 hours from onset to hospitalization $(32.75 \%$ vs $14.28 \%$ and $12.63 \%$ ).

Table 4. PIS among different antiplatelet groups NIHSS $>3$ points.

\begin{tabular}{llll}
\hline $\begin{array}{l}\text { Antiplatelet } \\
\text { program }\end{array}$ & Aspirin & $\begin{array}{l}\text { Routine } \\
\text { Clopidogrel }\end{array}$ & $\begin{array}{l}\text { Loading } \\
\text { Clopidogrel }\end{array}$ \\
\hline Without PIS & 18 & 40 & 53 \\
With PIS (\%) & $12(66.67 \%)$ & $10(20.00 \%)$ & $8(13.11 \%)$ \\
Total & 30 & 50 & 61 \\
\hline
\end{tabular}

This table shows that in the patients of NIHSS over 3 points, PIS is much higher in the group of aspirin while the clopidogrel group is much lower $(66.67 \%$ vs $20.0 \%$ and $13.11 \%)$.

Table 5. PIS among different groups of NIHSS $>3$ points.

\begin{tabular}{llll}
\hline NIHSS Group & With PIS (\%) & Without PIS & Total \\
\hline NIHSS $4-7$ & $26(16.56 \%)$ & 131 & 157 \\
NIHSS $8-15$ & $16(26.23 \%)$ & 45 & 61 \\
NIHSS $>15$ & $7(20.0 \%)$ & 28 & 35 \\
Total & $49(19.37 \%)$ & 204 & 253 \\
\hline
\end{tabular}

This table shows that the PIS of NIHSS within 7 potints is lower than those over 7 points. The average PIS percentage is $19.37 \%$ in patients of NIHSS over 3 points which is much higher than those within 3 points $(8.15 \%)$.

\subsection{Safety Endpoint Event}

Major Safe end event: 2cases. Both were Symptomatic intracranial hemorrhage of NIHSS increasing over 4 points and underwent aspirin and clopidogrel respectively.
Table 6. Secondary Safe end event among different antiplatelet groups.

\begin{tabular}{llll}
\hline Antiplatelet program & Aspirin & $\begin{array}{l}\text { Routine } \\
\text { Clopidogrel }\end{array}$ & $\begin{array}{l}\text { Loading } \\
\text { Clopidogrel }\end{array}$ \\
\hline No safe end & 125 & 191 & 156 \\
Secondary safe end event (N, \%) & $7(5.3 \%)$ & $14(6.83 \%)$ & $6(3.68 \%)$ \\
P & - & 0.572 & 0.534 \\
Total & 132 & 205 & 163 \\
\hline
\end{tabular}

This table shows that there's no obvious difference between aspirin and Clopidogrel groups in safe endpoint events.

\section{Discussion}

This is a retrospective study and the range of information is restricted in 7 days. The average of early recurrence or progression percentage is $9.33 \%$ when compared with other researches such as the CLASS-CHINA [7] in which the PIS in loading dosage group is $16.1 \%$ and the control group is $14.9 \%$. It may because of the lack of case recorded and short of studying time. Loading dosage of clopidogrel can rapidly achievement homeostasis state of drug and has been proved to be safe $[11,12]$.

PIS is most likely to occur in the patients within 24 hours from onset to hospitalization which prompt the first 24 hours is the most important for ischemic stroke patients. Although the PIS may be affected by multiple factors, time is always the most important. The sooner of treatment started, the fewer of PIS. But there's no recorded data of the time from onset or treatment to PIS and this need for further work and research. The criteria from onset to progression of time is not so clear and can range from 2 to 30 hours or even 7 days [13].

The PIS percentage is only $8.15 \%$ in patients of NIHSS within 3 points while the average PIS percentage is $19.37 \%$ in patients of NIHSS over 3 points which prompt the more severe of neurological deficit, the higher of PIS percentage. This may because of higher risk stratification of patients with higher NIHSS scores.

CHANCE study [1] proved the superiority of loading and combination of antiplatelet over aspirin alone in TIA (ABCD2 score $\geq 4$ points) and the infarction (NIHSS score $\leq 3$ points) patients. In our study there's no superiority of clopidogrel to aspirin in patients of NIHSS score $\leq 3$ points. Whether there'll be superiority of loading and combination of clopidogrel and aspirin to aspirin with different TOAST etiology of stroke for over 7 days or even 21 days need for further research.

Combined antiplatelet group stands for $54.7 \%(269 / 492)$ in patients of NIHSS $0-3$ points and $56.6 \%(454 / 802)$ in patients of NIHSS 0-7 points. There's a total of 489 cases (49\%) with combined antiplatelet treatment which means the standard operation process is insufficient and part of the patients with higher than 7 points of NIHSS underwent antiplatelet drug of more than one kind.

There's no obvious difference between aspirin and clopidogrel groups in safe endpoint events which prompt that loading dosage of clopidogrel is also safe in clinc and the PRESS-CHINA and CLASS-CHINA have proved the safety 
of loading clopidogrel.

\section{Conclusion}

Clopidogrel group was superior to aspirin in reducing early recurrence and deterioration within 24 hours, while NIHSS on admission was greater than 3 points and NIHSS score was $8-15$.

PIS has close relation to admission time after onset of clinc especially the first 24 hours is the most important for ischemic stroke patients which prompt once again time is brain. Clopidogrel is superior to aspirin in patients within 24 hours from onset to hospitalization. Besides, PIS has close relation to severity of clinc and the PIS in patints of NIHSS within 3 points is much lower than those over 3 points. In the patients of NIHSS over 3 points the clopidogrel group is superior to aspirin group.

There's no obvious difference between aspirin and clopidogrel groups in safe endpoint events and loading dosage of clopidogrel is as safe as the routine group and aspirin group.

\section{Acknowledgements}

"Ischemic Stroke" / "TIA" antiplatelet Therapy Clinical research funded by Xinlitai pharmaceutical industry.

\section{References}

[1] CAST: randomised placebo-controlled trial of early aspirin use in 20,000 patients with acute ischaemic stroke. CAST (Chinese Acute Stroke Trial) Collaborative Group. Lancet (London, England) 1997; 349: 1641-9.

[2] The International Stroke Trial (IST): a randomised trial of aspirin, subcutaneous heparin, both, or neither among 19435 patients with acute ischaemic stroke. International Stroke Trial Collaborative Group. Lancet (London, England) 1997; 349: 1569-81.

[3] A randomised, blinded, trial of clopidogrel versus aspirin in patients at risk of ischaemic events (CAPRIE). CAPRIE Steering Committee. Lancet (London, England) 1996; 348: 1329-39.
[4] Seadon S, Lang E. Clopidogrel with aspirin versus aspirin alone in prevention of stroke following transient ischemic attack or acute minor stroke. Cjem 2015; 17: 315-7.

[5] Derdeyn CP, Chimowitz MI, Lynn MJ et al. Aggressive medical treatment with or without stenting in high-risk patients with intracranial artery stenosis (SAMMPRIS): the final results of a randomised trial. Lancet (London, England) 2014; 383: 333-41.

[6] Johnston SC, Amarenco P. Ticagrelor versus Aspirin in Acute Stroke or Transient Ischemic Attack. The New England journal of medicine 2016; 375: 1395.

[7] Zhao Y, Yang W, Tan Z et al. Clopidogrel loading dose versus maintenance dose to treat patients with acute ischaemic stroke in China (CLASS-China): results from a prospective double-blind randomised clinical trial. Stroke and vascular neurology 2017; 2: 118-23.

[8] Meyer DM, Albright KC, Allison TA, Grotta JC. LOAD: a pilot study of the safety of loading of aspirin and clopidogrel in acute ischemic stroke and transient ischemic attack. Journal of stroke and cerebrovascular diseases: the official journal of National Stroke Association 2008; 17: 26-9.

[9] Wang Y, Pan Y, Zhao X et al. Clopidogrel With Aspirin in Acute Minor Stroke or Transient Ischemic Attack (CHANCE) Trial: One-Year Outcomes. Circulation 2015; 132: 40-6.

[10] Chinese Medical Association Neurology Society and Chinese Medical Association Neurology Branch of Cerebrovascular Disease. Guidelines for the diagnosis and treatment of Acute Ischemic Stroke in China 2014. Chinese Journal of Neurology 2015; 4: 246-257.

[11] Gurbel PA, Bliden KP, Hiatt BL, O'Connor CM. Clopidogrel for coronary stenting: response variability, drug resistance, and the effect of pretreatment platelet reactivity. Circulation 2003; 107: 2908-13.

[12] Leung LY, Albright KC, Boehme AK et al. Short-term bleeding events observed with clopidogrel loading in acute ischemic stroke patients. Journal of stroke and cerebrovascular diseases: the official journal of National Stroke Association 2013; 22: 1184-9.

[13] Birschel P, Ellul J, Barer D. Progressing stroke: towards an internationally agreed definition. Cerebrovascular diseases (Basel, Switzerland) 2004; 17: 242-52. 